\title{
Research on Rapid Product Design Based on WAVE Technology
}

\author{
Linzhen Zhou, Qingzhu Li \\ School of Mechanical Engineering, Yancheng Institute of \\ Technology, Yancheng, 224051, China
}

\begin{abstract}
Rapid design systems in existence mainly target at series development instead of product variant design. As an effort to solve this problem, this paper applies WAVE (What-if Alternative Value Engineer) to rapid product design. It elaborates on the basic theory and design method of WAVE and points out ways to integrate it with product rapid design system. Then using WAVE, it introduces knowledge expression, storage and deduction, the establishment of prototype library and the automatic updating of prototype model in rapid design system. Finally the system's validity is proved through a design case of modular machine tool.
\end{abstract}

Keywords-WAVE, rapid design, knowledge based engineering, modular machine tool.

\section{INTRODUCTION}

The rapid development of modern manufacturing industry imposes more stringent requirements on the structure and properties of products. To stand up to today's rapidly changing market, a company's ability of rapid product design is crucial for remaining successful. Product design work focuses on adaptive design and variant design in the actual production. How to use the advanced design methods, ideas and concepts, acquire and reuse design knowledge in order to develop new products to meet customer requirements as soon as possible has become a great challenge to modern companies.

The common practice of most existing product rapid design systems is to build digital models of each part before digital assembly. When some parts turn to be undesirable in the assembly, they have to be redesigned and reassembled. This drawback arises from the fact that these systems do not support the whole process from concept design to detailed design. Different from parametric modeling at part level, WAVE is a product parameterization technology. It runs the changes of concept design and detailed design through the entire product design process. When engineering parameters of a product are changed, the system will automatically update the product to part level according to design criteria saved in the control structure, so as to ensure design idea and integrity.

This paper applies WAVE technology to product rapid design system to realize rapid design of a series of products with variant structures. It elaborates on knowledge exploration and expression and key technologies to establish and implement inference engine and prototype database. Finally, this paper demonstrates the application of WAVE in rapid design system through a case design of a combined machine tool.

\section{WAVE TECHNOLOGY}

WAVE is a parameter design technology at product level. As an optimization engineering design of automatic inference, it provides a top-down full product design environment required in product design. It correlates the overall assembly design of product and detailed part design. Once a controlling parameter is changed, it will automatically update all parts related in accordance with the scheduled control structure, geometrical relationship and design criteria to ensure the integrity of product design.

In the actual project application, product design is usually top-down, that is, following the pattern of conceptual design - component design - part design. The WAVE control structure is similar to the product assembly structure: the upper layer contains and controls geometric object information of sub layer; the position and size of its geometric objects change correspondingly when the upper layer parameters are changed. As the sub layer geometric objects are related to the upper layer geometric objects, the position and size of the sub layer geometric objects are also updated. Hence it realizes the authentic top-down design. A typical control structure has two layers: automatic inference layer and isolation layer as shown in Fig. 1.

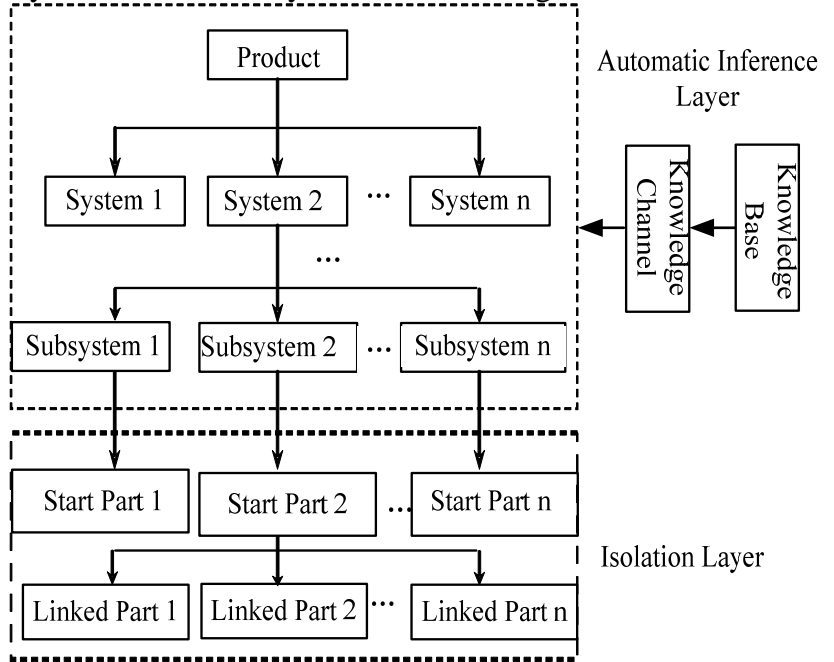

Figure 1. The composition of control structure

Automatic inference layer contains the product definition information and product demand standards as well as the relationship standards between various subsystems. Isolation layer contains starting components and connecting components. Starting components bring about the connection of actual detailed assembly or conceptual design assembly, and serve as the starting point 
of one or more connecting components. Connecting components contain the details of geometric objects, which are used as the components of product assembly. Starting components restrict connecting components by a "control cable", so any change occurred at starting components will be passed to connecting components.

\section{METHODS}

\section{A. Interpart modeling}

Interpart modeling is a method to establish the parameterization and correlation within parts or between parts when the geometric objects participating in the connection are in the same part or in different parts of the same assembly structure. It offers top-down parameters control through sharing geometric information of parts with sub layer or same layer.

WAVE provides geometric coupling, copying geometry and other tools to achieve interpart modeling. Geometric coupling allows copying geometric objects to the current working parts from any part of related assembly part and finally correlates these geometric objects to father geometric objects, whose change will cause the updating of correlated geometric objects. It can be used to establish and identify the new features of geometric objects in the working parts. Copying geometry can copy and relate geometric objects in the current working parts to other components. As WAVE geometric coupling, when geometric objects defined in father parts are changed, all geometric objects extracted from other parts will update automatically. Copying geometry is mostly used in topdown design, whose main function is to release the geometric objects in working parts files to other parts files for further modeling. In addition, the upper layer geometries can be released to the sub layer parts files in the option of new-built layer to realize the sharing of geometries between parts.

\section{B. Top-down design.}

Top-down design allows designers to define the overall parameters, periphery contour size and the main positioning parameters of parts in the upper layer of assembly structure. These parameters are passed to correlated parts of the sub layer. Detailed design is constructed at the bottom layer. So when the upper layer part design is changed, designers can update components design automatically. The key parameter can be modified on the top layer and passed down to the bottom details. Since conceptual design data and detailed design data are defined in different parts, detailed design can be put into effect before conceptual design is completed. Therefore, it provides a sound foundation from conceptual design to parts detailed design and concurrent product design.

Top-down design is suitable for moderately complex products, which uses datum planes or sketches to control the basic shape, size and position of assembly of all parts in the whole product. In the top-down design, control structure and the files of parts are in the same assembly file, and the location and shape defined by control structure are copied to subsystems and components. When the key engineering parameters are changed, related subsystems and components will be updated automatically.

\section{Design based system engineering.}

Design based system engineering adopts a "control structure" similar to assembly structure to define the overall assembly structure of product, and then divides the overall structure into several subsystems (or sub assembly), which are used for grouping and concurrent design. Difference of WAVE system engineering from the top-down design is it defines the control structure as an independent assembly file, which is known as product template. To be specific, the control structure is a set of design variables, which controls design components of defined assembly structure and design history. It can be borrowed for later similar product design. Control structure possesses information about control, function and configuration of product at the bottom layer of the tree structure, by which a product can be divided into different regions associated with each other. Designers only need to execute the corresponding design work in each region. When the control structure is changed, all related parts will be updated in accordance with design scheme automatically.

Design based system engineering is suitable for large complex product design since it supports collaborative team design and concurrent design. It solves complex product design problem by introducing "control structure", which comprises general control parameters and provides the restrain framework for further assembly design or detailed design by expressing the requirements of overall design through some simple geometries and the positions of key parts.

\section{APPLICATION}

\section{A. Representation and storage of product structure knowledge}

Product structure knowledge mainly reflects geometric structure, size of parts and space constraints, assembly relations and geometry relations between parts. Structure knowledge of modular machine tools mainly includes the overall size of product shape, the assembly size and mutual position relationships of different parts. With WAVE technology, this system expresses structure knowledge of modular machine tools in sketches and datum planes as its framework through the ways.

The system controls topological structure of parts through sketches, and saves them in product control structure in the form of geometry elements. For parts and relationships between parts whose structure, position and direction all may change when model is updated, they can be expressed with the position relationships of datum planes, which can restrict the location of a specific part in the overall product structure.

\section{B. Realization of inference engine.}

The configuration rules are the key to utilize knowledge base to configure product. They could be simple or complex. 
Common configuration rules are variable rules, validity rules and delivery status rules.

Inference engine is the core to realize automatic design. This system uses forward reasoning. The designer types in product requirements such as channel width and channel depth through man-machine interface. Inference engine will match the fact with the premise part of each rule in knowledge base successively. If the matching succeeds, the conclusion part of this rule will be added to fact product design process as new facts. Since the design is top-down, these conclusions will generate new facts with the design going in-depth until all parts are designed.

\section{Updating of prototype model.}

The system clones the prototype model with assembly cloning technology and loads the cloned 3D model to product model. The cloned 3D model inherits all information of prototype model. So the 3D model loaded to product model contains product structure knowledge reflecting design idea and geometric relationships between parts.

Though 3D model which is cloned and loaded to product model contains product structure knowledge reflecting geometric relationship between parts, the geometries reflecting product structure knowledge are broken. This system reconnects geometries through restoring association features, that is, through recovering geometric relationship between sub-model and father model to realize geometric control of new loaded 3D model for product control structure. Because product control structure is customized according to the engineering parameters requirements by designers, the new loaded model will be updated according to the input engineering parameters automatically.

\section{IMPLEMENTATION}

Here is an instance to illustrate the application of WAVE in rapid modular machine tool design system. Modular machine tool is an efficient special machine tool which is constituted on the basis of standard generalpurpose components and complemented with some special parts. It is often designed according to the characteristics of parts being machined. With changeable structure, its design is complex. Rapid modular machine tool design system can greatly shorten the development cycle. The application of WAVE technology in the design system can achieve development association between the overall design and detailed design of every component and auxiliary system.

According to the "top-down" design idea, overall design of modular machine tool can be divided into the following subsystems: fixture control system, configuration control system, multi axle box control system, cutter connecting rod control system, and parts to machine. The parts to machine are added to in forms of components. The quotation of parts and generation of assembly contents of all levels are through WAVE. According to the preset processing technology and configuration form information, overall control structure is built using geometry linker and correlation constraints are established between subsystems.
Fig. 2 is the control structure defined by datum planes of each subsystem of modular machine tool relative to parts to machine.

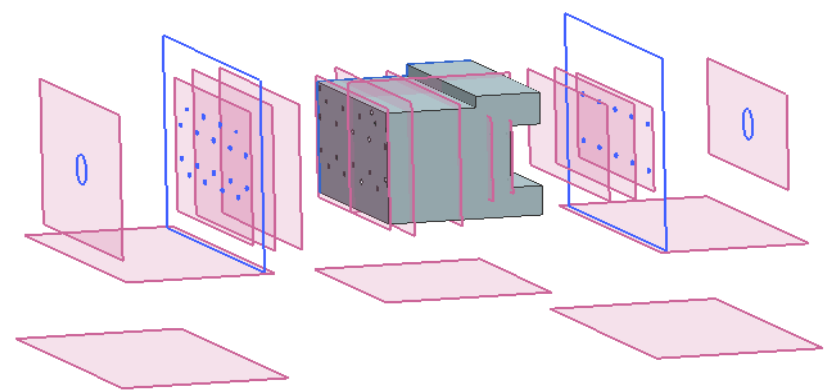

Figure 2. The control structure of each subsystem of modular machine tool relative to parts to machine

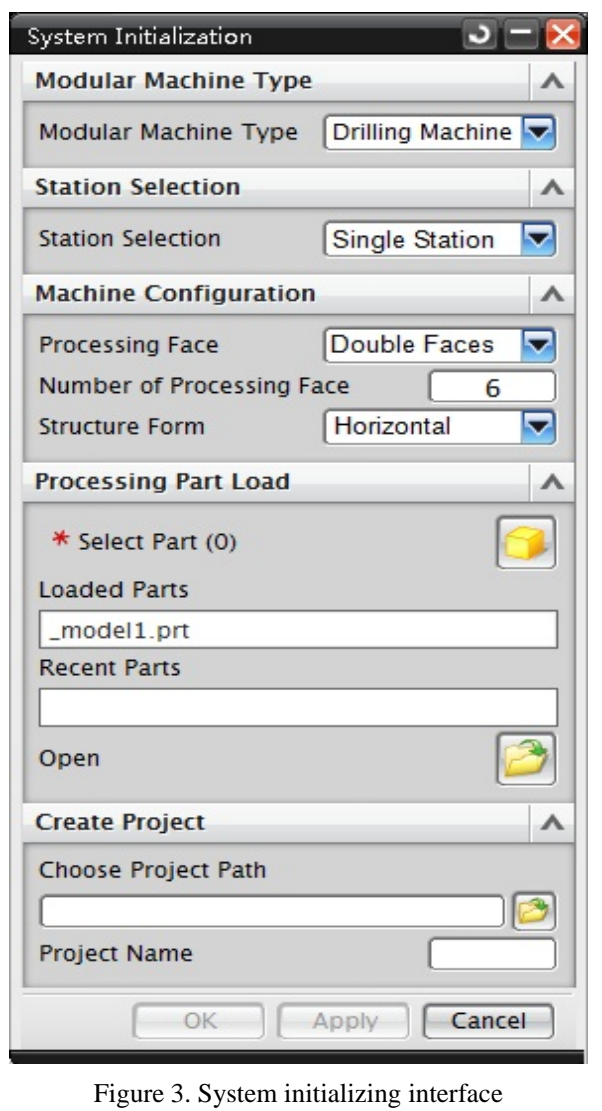

The system is developed in Windows XP environment, and uses object-oriented $\mathrm{CH}$ as its development language on .NET development platform. The customization is carried on NX of Siemens Company, a new generation digital product development system. User interface design is customized through BlockStyler. Menu and toolbar are created through MenuScript. Journaling and NX Open are employed in design knowledge capture and system development. The system will load MenuScript file automatically and display modular machine tool design menu on NX system menu bar. The main interface of 
modular machine tool design system is shown as Fig.3. Designers conduct overall design, fixture design, multi axle box design and other designs of modular machine tool by process wizard. The system will assemble and generate 3D digital model of modular machine tool automatically after the design is finished. Fig.4 is a 3D digital model of doublesided drilling horizontal modular machine tool.

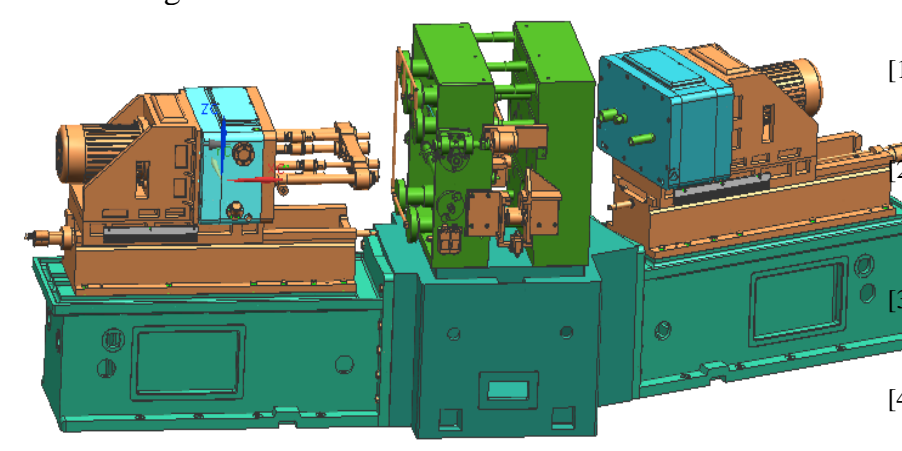

Figure 4. Digital prototype of the modular machine tool

\section{CONCLUSIONS}

The paper combines practical technology of knowledge engineering with WAVE. It solves knowledge exploration and expression in rapid design system of variant products and the key technology to build and implement inference engine and prototype database. It applies WAVE in modular machine tool design and develops a rapid design system as is shown in Fig.3 and Fig.4. Designers only need to input a few parameters. The system will generate new 3D digital models of a series of products under the intelligent guidance of inference machine. It helps companies to accumulate and inherit design experience and provides reliable technical guarantee for the product design quality. Hence it considerably shortens the design cycle, reduces design cost and gives the company an edge in the competitive market.

\section{ACKNOWLEDGEMENTS}

The research work was supported by six talent summit foundation of Jiangsu Province, China (No. ZBZZ-038).

\section{REFERENCES}

[1] Lu, C.M. \& Zhang, L.B. A research on rapid product configuration design method based on design templates, Computer Integrated Manufacturing System, 36(10), pp.425-430, 2009.

2] Li, X.H., Liu, R.P., Gao, X.M. \& Hong, J. Studies on rule and casebased reasoning of product optimization configuration for CNC machine tools, Mechanical Science and Technology for Aerospace Engineering, 56(7), pp.1533-1542, 2011.

Zhao, J., Fu, L., Ma, W. \& Chen A. Top-down Design of Sweeper's Plate Brush System Based on NX/WAVE, Mechanical Engineer, 23(3), pp.146-148, 2014.

[4] Wang, X.H., Liao, W.H. \& Liu, D.F. A knowledge-based study on CAD system of overall modular machine tool design, Modular machine tools and automation technology, 18(2) , pp. 45-47, 2002.

[5] Ren, X.Z., Li, C.M., Su, J.X. \& Du, X.S. Modular design of modular machine tool based on UG, Tractors and farm vehicles, 34(2):28-29, 2007.

[6] Padayachee, J. \& Bright, G. Modular machine tools: Design and barriers to industrial implementation, Journal of Manufacturing Systems, 31(2), pp.92-102, 2012.

[7] Lorenzer, T., Weikert, S., Bossoni, S. \& Wegener, K. Modeling and evaluation tool for supporting decisions on the design of reconfigurable machine tools, Journal of Manufacturing Systems, 26(3) , pp.167-177, 2007.

[8] Daisuke, K., Thomas, L., Sascha, W. \& Konrad, W. Evaluation of modeling approaches for machine tool design, Precision Engineering, 34(3), pp.399-407, 2010.

[9] Liu, X.H., Li, Q., Liu, Y.H. \& Yin, J. Development of a rapid design system for aerial work truck subframe with UG secondary development framework, Procedia Engineering, 45(2) , pp.2961-2965, 2011. 